\title{
Post-COVID Syndrome (MIS-C) with Refractory Status Epilepticus
}

\author{
Haripal Kashyap ${ }^{1} \cdot$ Vineetranjan Gupta $^{1} \cdot$ Arvind Gupta $^{1} \cdot$ Tarun Gupta $^{1} \cdot$ Seshendra Sharma ${ }^{1} \cdot$ Shreyansh Valjiyani $^{1}$
}

Received: 4 January 2021 / Accepted: 8 March 2021 / Published online: 21 April 2021

(C) Dr. K C Chaudhuri Foundation 2021

To the Editor: Multisystem inflammatory syndrome in children (MIS-C) has varied symptoms that affect several organs and systems in body [1]. We present this case with refractory status epilepticus to highlight the neurological complication of this syndrome with an aim to spread awareness of the clinical manifestations of this syndrome among the treating clinicians.

A 7-mo-old male child presented with complaints of fever with maculopapular rash with 1 episode of abnormal movement at the onset of fever of 2-d duration with conjunctival congestion. Post admission, the child had 1 episode of generalized tonic clonic seizure, which was not controlled by 1st and 2nd line antiepileptic drugs; hence was intubated and started on injection midazolam infusion. Investigation showed raised CRP level of $8.73 \mathrm{mg} / \mathrm{dL}$. There was positive history of COVID infection in family members $20 \mathrm{~d}$ back; hence, in view of that COVID-19, IgG antibody level was done in this child, which came positive with value of $4.74 \mathrm{~s} / \mathrm{co}$ (normal value $<1.4 \mathrm{~s} / \mathrm{co}$ ). Rest of the fever workup including cerebrospinal fluid (CSF) examination and neuroimaging was normal, which ruled out the causes of status epilepticus. In view of positive COVID-19 antibody, inflammatory markers were sent, which showed raised NT Pro-BNP - 2536, D-dimer - 1839, Ferritin 86.26, hence a diagnosis of post-COVID syndrome (MIS-C) was made as per WHO criteria [1].

The child was treated with pulse methylprednisolone therapy but in view of rising trend of inflammatory marker and persistent fever, intravenous immunoglobulin (IVIG) was given $(2 \mathrm{~g} / \mathrm{kg})$. The child improved over next $48 \mathrm{~h}$ and was discharged.
The hallmark of MIS-C is widespread inflammation across multiple organ systems [2]. Left untreated, this inflammation can cause long-term morbidity and increased mortality [2]. Medication commonly used in children with MIS-C includes steroids, IVIG, low-molecular-weight heparin (LMWH), and aspirin. In refractory MIS-C cases, other treatment options are tocilizumab, anakinra, and infliximab [3]. Prompt diagnosis with institution of appropriate therapy is the key to favorable outcome in MIS-C [4].

\section{Declarations}

Conflict of Interest None.

\section{References}

1. https://www.who.int/news-room/commentaries/detail/multisysteminflammatory-syndrome-in-children-and-adolescents-with-covid19. Accessed Dec 2020.

2. Multisystem Inflammatory syndrome in children. Available at: https://www.yalemedicine.org/conditions/multisysteminfalammatorysyndrome-inchildren-mis-c. Accessed 20 Dec 2020.

3. Jones VG, Mills M, Suarez D, et al. COVID-19 and Kawasaki disease: novel virus and novel case. Hosp Pediatr. 2020;10:537-40.

4. CDC COVID-19 Response Team. Coronavirus disease 2019 in children - United States, February 12-April 2, 2020. MMWR Morb Mortal Wkly Rep. 2020;69(14):422-6.

Publisher's Note Springer Nature remains neutral with regard to jurisdictional claims in published maps and institutional affiliations.

Vineetranjan Gupta

myvineet1689@gmail.com

1 Department of Pediatrics, Asian Institute of Medical Sciences, Faridabad, Delhi NCR 121001, India 